\title{
Piezoelectric resonator arrays for tunable acoustic waveguides and metamaterials
}

\author{
Filippo Casadei, ${ }^{1, a)}$ Tommaso Delpero, ${ }^{2, a), b)}$ Andrea Bergamini, ${ }^{3}$ Paolo Ermanni, ${ }^{2}$ \\ and Massimo Ruzzene ${ }^{1}$ \\ ${ }^{1}$ School of Aerospace Engineering, Georgia Institute of Technology, Atlanta, Georgia 30332, USA \\ ${ }^{2}$ Centre of Structure Technologies, ETH, Zurich, Switzerland \\ ${ }^{3}$ Swiss Federal Laboratories for Materials Science and Technology, EMPA, Dübendorf, Switzerland
}

(Received 30 April 2012; accepted 15 August 2012; published online 19 September 2012)

\begin{abstract}
One of the outstanding challenges in phononic crystals and acoustic metamaterials development is the ability to tune their performance without requiring structural modifications. We report on the experimental demonstration of a tunable acoustic waveguide implemented within a two-dimensional phononic plate. The waveguide is equipped with a periodic array of piezoelectric transducers which are shunted through passive inductive circuits. The resonance characteristics of the shunts lead to strong attenuation and to negative group velocities at frequencies defined by the circuits' inductance. The proposed waveguide illustrates the concept of a controllable acoustic logic port or of an acoustic metamaterial with tunable dispersion characteristics. (c) 2012 American Institute of Physics. [http://dx.doi.org/10.1063/1.4752468]
\end{abstract}

\section{INTRODUCTION}

Phononic crystals ${ }^{1-3}$ and acoustic metamaterials ${ }^{4-6}$ have attracted significant interest for the development of devices which exhibit, among others, acoustic super-lensing, superfocusing, and cloaking characteristics ${ }^{7-9}$ that may lead to a large number of potential applications. Their interesting properties rely on the ability to tailor the propagation of acoustic waves through the generation of acoustic bandgaps. ${ }^{10-15}$

Bandgaps are the result of wave scattering at periodic impedance mismatch zones (Bragg scattering) ${ }^{3}$ or are generated by resonating units within the medium. ${ }^{4,16,17}$ While Bragg scattering bandgaps occur at wavelengths of the order of the unit cell size, local resonances produce frequency attenuation regions which are independent of the lattice constant defining the spatial periodicity of the medium. For this reason, locally resonant materials are of particular interest due to their ability to generate low frequency attenuation and the possibility of providing the medium with unusual mechanical properties at long wavelengths. The latter is the main objective in the study and development of acoustic metamaterials, ${ }^{4,5}$ while Bragg scattering is often exploited in phononic crystals designed to filter, localize, and guide acoustic waves. ${ }^{10,15}$

Locally resonating acoustic metamaterials have been implemented by considering single ${ }^{18}$ and multiple degrees of freedom resonating units such as soft inclusions periodically dispersed in a hard material matrix ${ }^{4,19-25}$ or periodic arrays of tuned Helmoltz resonators in an acoustic waveguide. 5,6 While most of the proposed metamaterial configurations operate at fixed frequency ranges, recent investigations have considered the use of large deformations ${ }^{26}$ or electro/magneto mechanical couplings ${ }^{27,28}$ as viable solutions to achieve tunable bandgaps and equivalent mechanical properties.

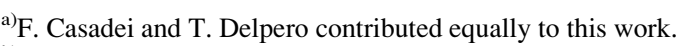

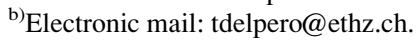

This paper reports on a new tunable waveguide configuration, whereby Bragg scattering bandgaps are combined with piezoelectric resonators to confine and control the propagation of elastic waves in a phononic crystal plate. ${ }^{14}$ The waveguide consists of a periodic array of cylindrical stubs bonded to the plate surface. The stubs produce a large frequency bandgap that confines the propagation to the waveguide. A second array of piezoelectric resonators is added to the vertical portion of the waveguide for wave transmission control. The resonating characteristics of the shunted piezos lead to strong wave attenuation at the tuning frequency and provide the waveguide with resonating mechanical properties, which lead to negative group velocities. From this perspective, the waveguide can be considered as an example of a tunable acoustic metamaterial, with equivalent properties defined by the electro-mechanical resonators.

\section{EXPERIMENTAL METHODS}

\section{A. Setup and materials}

The considered phononic crystal consists of a thin aluminum plate $(1 \mathrm{~mm}$ thick) with a periodic arrangement of surface-bonded cylindrical stubs of diameter $d=7 \mathrm{~mm}$ and height $h=10 \mathrm{~mm}$. The stubs are made of aluminum and are arranged in a square lattice of constant $a=10 \mathrm{~mm}$. Standard epoxy glue is used to bond the stubs to the plate surface.

An L-shaped waveguide is realized within a $17 \times 17$ lattice by removing parts of one row and one column of stubs as shown in Fig. 1(a). The vertical portion of the channel is equipped with a periodic array of $8 \times 1$ piezo-ceramic $(\mathrm{PZT})$ disks (Steminc SMD10T04F: diameter $d_{p}=10 \mathrm{~mm}$, capacity $\left.C_{p}=1.8 \mathrm{nF}\right)$. Each transducer is independently shunted through an inductive circuit realized by means of passive axial inductors mounted on a conventional breadboard configuration. The considered resonating unit is schematically shown in Fig. 1(b), where the resistive element (R) accounts for any electrical loss introduced by wiring and connections. 


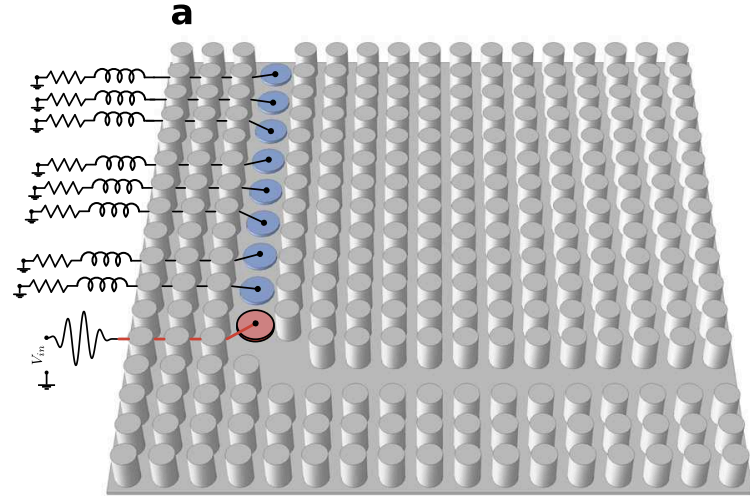

b

shunting impedance

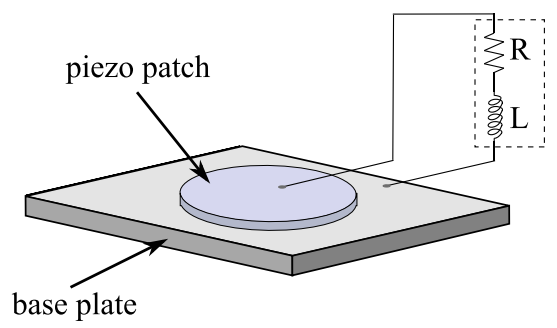

FIG. 1. New concept of tunable waveguide with electrical resonating units. (a) Illustration of the phononic crystal plate with the cylindrical stubs and the L-shaped waveguide. The vertical portion of the waveguide is equipped with a periodic arrangement of piezoelectric discs. Eight transducers are independently shunted with an inductive circuit, while the one located near the bend (in red) is used for exciting the structure. (b) Schematic model of the building block of the vertical channel. The combination between the capacity of the piezo and the electrical circuit creates the resonating unit that generates the tunable bandgap.

\section{B. Data processing}

The wave propagation properties of the structure are characterized through two-dimensional wavefield measurements, recorded by a scanning laser vibrometer (Politec PSV-400). Measurements are conducted over a grid of $140 \times 140$ scan points with a spatial resolution of $1 \mathrm{~mm}$ in the horizontal and vertical directions. At each grid point, the laser measures the time history of the component of velocity $w\left(x_{i}, y_{j}, t\right) i, j=1 \ldots 140$ normal to the plate surface. Each time history includes 512 samples and is recorded at a sampling frequency of $1.28 \mathrm{MHz}$.

The waves are excited by a piezoelectric transducer located near the bend in the L-shaped waveguide (depicted in red in Fig. 1(a). The transducer is driven by an input signal consisting of a four-cycle tone burst centered at $100 \mathrm{kHz}$, generated by a waveform generator (Agilent $33220 \mathrm{~A}-20 \mathrm{MHz}$ ), and amplified by a piezo driver amplifier (Trek PZD 350).

The measured data are analyzed through spatial and temporal Fourier transforms to eliminate disturbances from boundary reflections and to obtain direct estimates of real and imaginary parts of the wavenumber. This data processing starts with a frequency-wavenumber filtering ${ }^{29}$ to remove boundary reflections. Next, the response recorded in the waveguide is spatially averaged through the width

$$
\hat{w}(\ell, \omega)=\frac{1}{N_{\ell}} \sum_{i, j \in \ell} \hat{w}\left(x_{i}, y_{j}, \omega\right),
$$

where $\ell$ denotes a line across the width of the channel comprised of $N_{\ell}$ scan points. A spatial Fourier transform along the length of the channel leads to the frequency-wavenumber representation of the averaged response $(\hat{W}(k, \omega)=\mathcal{F}[\hat{w}(\ell, \omega)])$, whose amplitude is represented by the contours in Figs. 4(a) and 4(d) and discussed in Sec. III. The averaged waveguide response $\hat{w}(\ell, \omega)$ is further processed to obtain estimates of dispersion, defined by the variation of real and imaginary part of the wavenumber as a function of frequency. At each frequency, real and imaginary part of the wavenumber are, respectively, evaluated from the spatial phase change and spatial amplitude decay of the averaged response. ${ }^{28}$

\section{RESULTS AND DISCUSSION}

The transmission characteristics and the tunable properties of the waveguide are evaluated through the analysis of two-dimensional wavefield measurements recorded using a scanning Laser Doppler vibrometer on the back surface of the stubbed plate. The considered phonic plate, originally proposed by Wu et al., ${ }^{14}$ is characterized by a Bragg-type
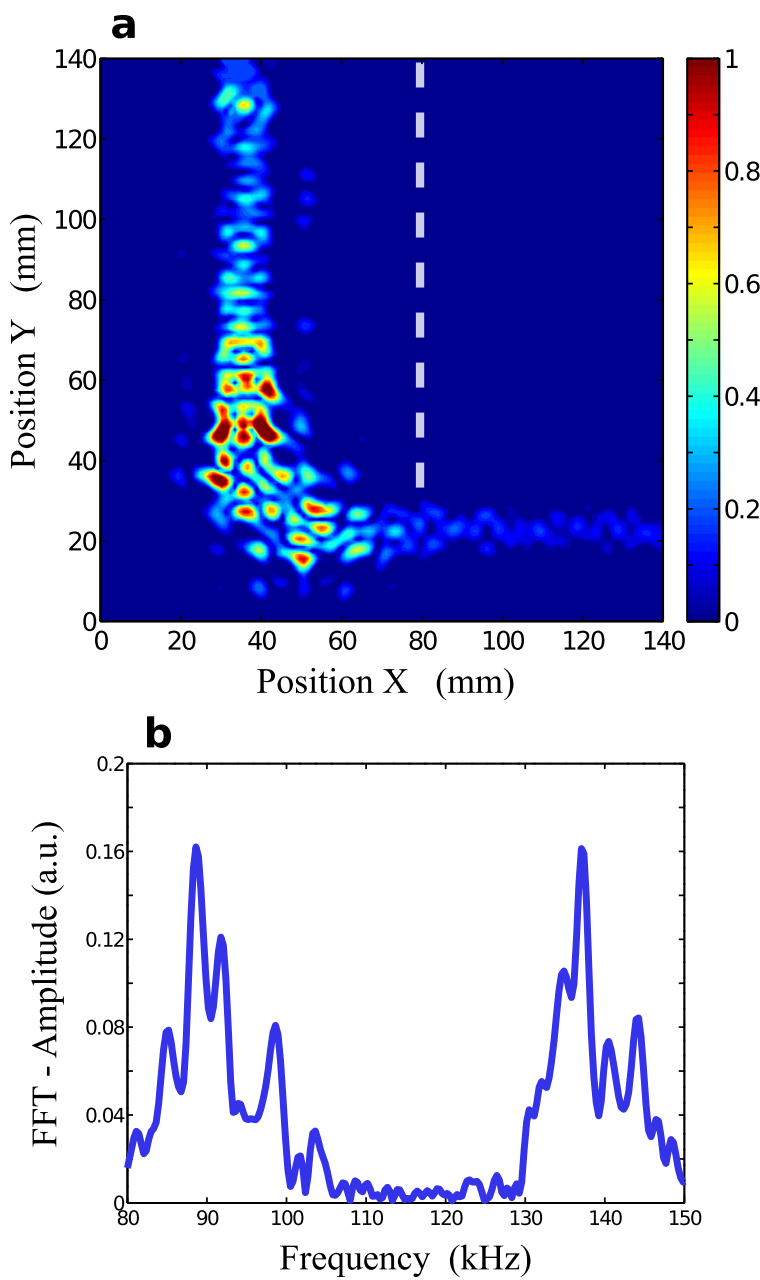

FIG. 2. Full wavefield measurements demonstrating the waveguiding capability of the phononic crystal plate. (a) Amplitude of the measured wavefield inside the Bragg-type bandgap at $117 \mathrm{kHz}$. Note that the presence of the piezoelectric discs in open-circuit configuration does not affect the wave transmission along the vertical channel. (b) The frequency spectrum of the wavefield averaged along the white dashed line in a clearly shows the extent of the bandgap between $100 \mathrm{kHz}$ and $130 \mathrm{kHz}$ generated by the stubbs. 


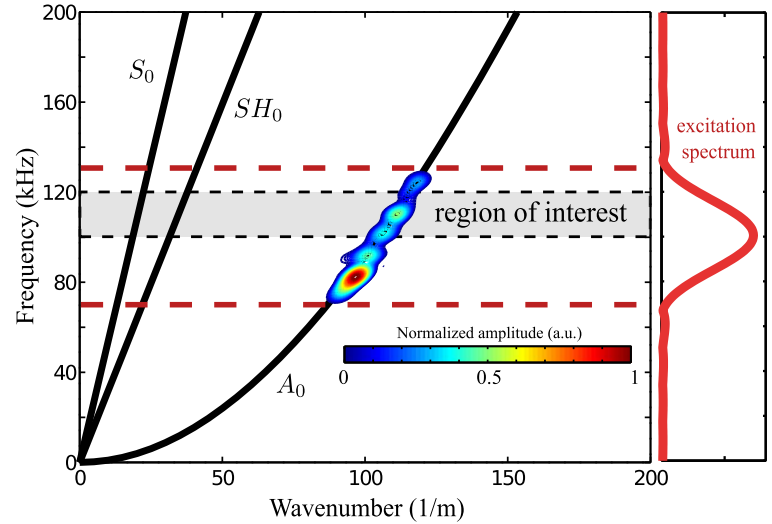

FIG. 3. Measured and calculated dispersion properties of the vertical channel with open circuits. The structure is excited in a frequency range between $70 \mathrm{kHz}$ and $130 \mathrm{kHz}$. The measured response along the vertical channel is processed and represented with contour lines in the frequency-wavenumber domain. The black solid lines represent the theoretical dispersion curves computed for a uniform $1 \mathrm{~mm}$ thick aluminum plate.

bandgap between 100 and $130 \mathrm{kHz} .{ }^{15}$ This frequency range is defined by the material and geometry of plate and stubs and cannot be changed after the system is assembled. Fig. 2(a) shows that the system behaves as an effective waveguide at frequencies inside the bandgap region illustrated in Fig. 2(b).

The resonant shunted piezos located in the vertical channel feature an effective frequency-dependent elastic Young's modulus $E_{S H}$ given by ${ }^{30}$

$$
E_{S H}(\omega)=E_{p}\left(1-\frac{k_{31}^{2}}{1+i \omega C_{p}^{\epsilon}(R+i \omega L)}\right)
$$

where $E_{p}$ is the Young's modulus of the piezo, $k_{31}$ is the electromechanical coupling coefficient of the piezoelectric disk, and $C_{p}^{\epsilon}$ is its electrical capacitance at constant strain. Also, $L$ is the shunting inductance and $R$ is the resistive element.

A mechanical strain applied to the piezo disk produces a voltage at its electrodes which is discharged through the electrical impedance defined by the series of the piezoelectric transducer capacitance $C_{p}$, the shunting inductance $L$, and the resistive contributions quantified by $R$. The piezoelectric disk then reacts on the plate by applying a force proportional to the produced voltage. The efficiency of the electro-mechanical coupling is defined by the dynamics of the system, which is dominated by the resonance of the shunting circuit. Such resonant characteristics are reflected in the expression of the equivalent Young's modulus given in Eq. (2) and lead to a behavior equivalent to a mechanical vibration absorber tuned at the approximate frequency $\omega_{T} \approx \sqrt{\frac{1}{L C_{p}}}($ for $R \approx 0$ ).

Of note is the fact that away from the resonance conditions or for the case of open shunting circuits, i.e., for $L, R \rightarrow \infty$, the contribution of the piezo disks to the overall stiffness of the waveguide appears as negligible. Therefore, the added stiffness corresponding to the array of disks is not sufficient to generate Bragg-scattering within the frequency
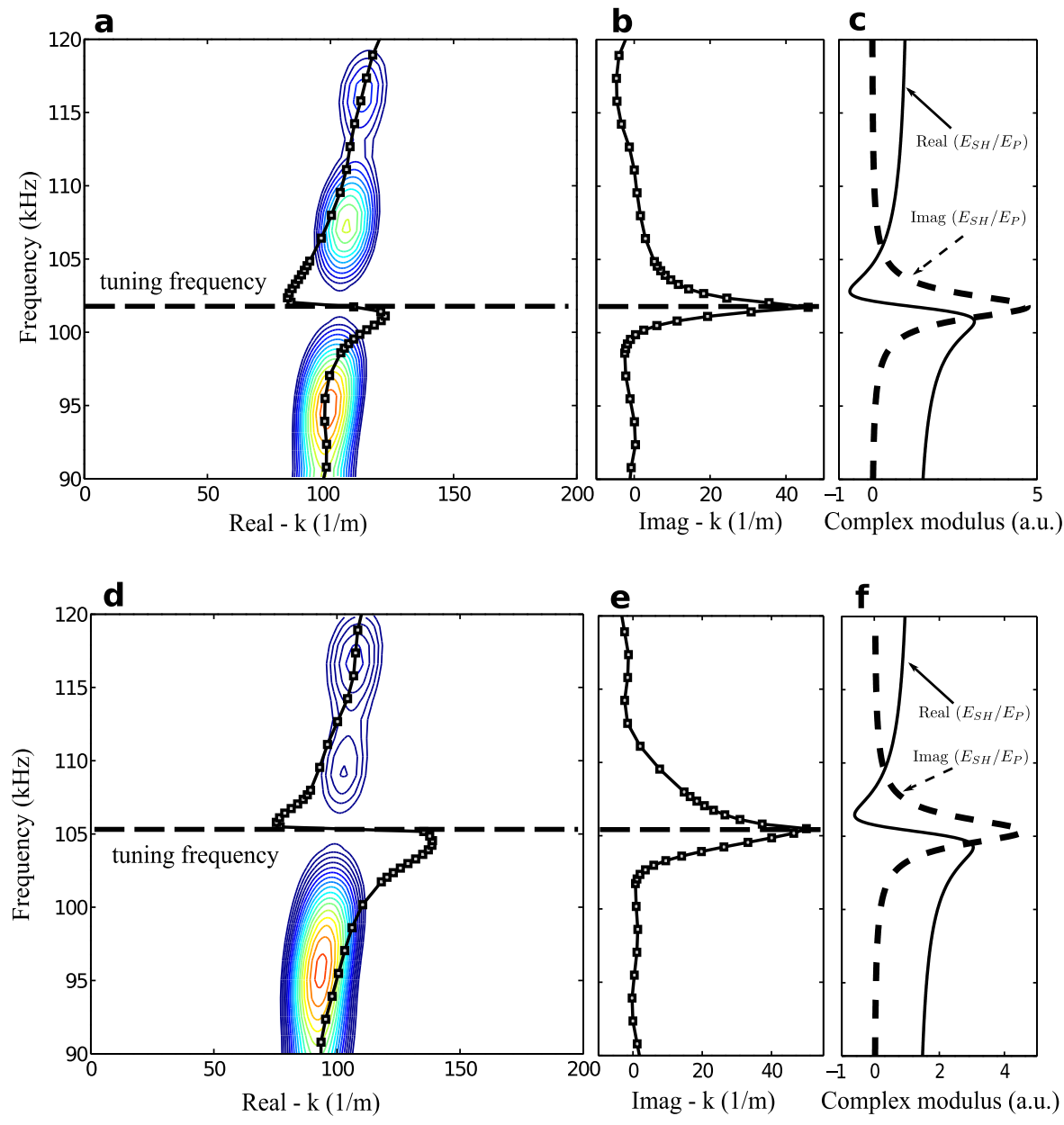

FIG. 4. Experimental evidence of the tunable resonant-type bandgap. (a) The contour lines reveal the presence of a bandgap in the response measured in the vertical channel with shunts tuned with $L=1890 \mu H$. The black line displays the estimated real part of the wavenumber and clearly shows the back-bending of the dispersion curve. (b) Estimated imaginary part of the wavenumber indicating the strong attenuation at the tuning frequency. (c) Real and Imaginary part of the effective dynamic modulus of the resonating units. (d)-(f) Same results as in (a), (b), and (c) but obtained for a different tuning frequency of the electrical shunts $(L=1560 \mu \mathrm{H})$ show the tunability of the bandgap. 
range of interest, so that waves propagate almost undisturbed within the channel.

The two-dimensional map of the wavefield recorded with open circuits shown in Fig. 2(a) clearly illustrates how the wave transmission within the channel is not altered by the presence of the piezo disks. Also, Fig. 3(a) shows the contour of the frequency/wavenumber domain representation of the wavefield recorded along the vertical waveguide. The contours are overlapped to the theoretical dispersion branch of the first antisymmetric $\left(A_{0}\right)$ Lamb wave mode for the considered plate. The close match between the contours and the theoretical dispersion, and the continuous frequency/wavenumber distribution defined by the contours, suggest how the behavior of the waveguide closely resembles that of a plane plate of the thickness considered in the experiments and that the presence of the piezos has little effect on wave transmission.

Shunting of the piezos through the electrical networks produces a resonant, complex modulus, which in turn generates a frequency region of strong attenuation centered at $\omega_{T}$. The tunable properties of the waveguide are illustrated by wavefield measurements with circuits designed to resonate at different frequencies. Specifically, two sets of inductors $\left(L_{1}=1890 \mu \mathrm{H}, L_{2}=1560 \mu \mathrm{H}\right)$ are used to tune the resonating units within the bandgap of the stubbed plate. In each case, contours of the frequency/wavenumber representation of the response, shown in Figs. 4(a) and 4(d), respectively, indicate that the resulting medium features a bandgap centered at the tuning frequencies.

The measured data also allow the experimental evaluation of real and imaginary part of the wavenumber, also known as attenuation and propagation constant. The real component of the wavenumber, overlapped as a solid line with square markers to the contour plots in Figs. 4(a) and $4(d)$, reveals the back-bending of the dispersion curve typical of internally resonating metamaterials. In this range of frequencies, elastic waves travel with a negative group velocity, antiparallel to the phase velocity. Also, the imaginary part, shown in Figs. 4(b) and 4(e), exhibits a sharp peak of attenuation in a small range of frequencies centered at the resonance of the electrical circuit $\omega_{T}$.

The resulting attenuation properties of the waveguide are responsible for the onset of the tunable bandgap and can be correlated to the frequency variation of the effective modulus of the resonating units as shown in Figs. 4(c) and 4(e). Furthermore, the back-bending characteristics of the dispersion curves and the associated negative group velocity confirm the interpretation of the proposed waveguide as a tunable, internally resonating metamaterial.

\section{CONCLUSIONS}

The experiments presented herein demonstrate the possibility of implementing an elastic waveguide with tunable dispersion properties. In conventional phononic crystals, acoustic waves are diffracted only at wavelengths of the order of the lattice constant. In contrast, the proposed concept allows controlling the dispersion relations of the system so that bandgaps are generated at selected frequency ranges without modifying the lattice constants of the crystal. This phenomenon is also independent of the spatial periodicity of the resonating units and is controlled by the effective frequency-dependent modulus of the shunted piezoelectric transducers. The resulting metamaterial therefore behaves as a homogenized medium with tunable attenuation and resonant properties.

The proposed concept suggests novel opportunities in several fields of engineering and material science for the design of tunable acoustic metamaterials, acoustic switches, and logic ports, which can be actively controlled through external stimuli, and for applications which include vibration isolation and noise attenuation, as well as wave guiding, localization, and filtering.

The multi-field coupling is here identified as the enabling mechanism for the generation of tunable internal resonances and the achievement of unusual wave mechanics. Although in the present study the mechanism has been demonstrated through a simple experimental apparatus, this research opens a new frontier for the development of a novel class of materials that exploit different forms of energy and multi-field coupling for the achievement of tunable mechanical properties.

\section{ACKNOWLEDGMENTS}

The work was partially conducted during M. Ruzzene's visit as a visiting professor in the Center of Structures Technology of ETH and was also partially funded by grant from the National Science Foundation (CMMI Grant No. 0926776), monitored by Dr. E. Misawa.

The authors also thank L. Di Lillo at the ETH Zurich for the useful discussions.

${ }^{1}$ M. Sigalas and E. Economou, "Band structure of elastic waves in two dimensional systems," Solid State Commun. 86, 141-143 (1993).

${ }^{2}$ R. Martinez-Sala, J. Sancho, J. Sanchez, V. Gómez, J. Llinares, and F. Meseguer, "Sound attenuation by sculpture," Nature (London) 378(6654), 241 (1995).

${ }^{3}$ W. Cheng, J. Wang, U. Jonas, G. Fytas, and N. Stefanou, "Observation and tuning of hypersonic bandgaps in colloidal crystals," Nat. Mater. 5, 830-836 (2006).

${ }^{4}$ Z. Liu, X. Zhang, Y. Mao, Y. Zhu, Z. Yang, C. Chan, and P. Sheng, "Locally resonant sonic materials," Science 289, 1734 (2000).

${ }^{5}$ N. Fang, D. Xi, J. Xu, M. Ambati, W. Srituravanich, C. Sun, and X. Zhang, "Ultrasonic metamaterials with negative modulus," Nat. Mater. 5, 452-456 (2006)

${ }^{6}$ Y. Cheng, J. Xu, and X. Liu, "One-dimensional structured ultrasonic metamaterials with simultaneously negative dynamic density and modulus," Phys. Rev. B 77, 045134 (2008).

${ }^{7}$ J. Li, L. Fok, X. Yin, G. Bartal, and X. Zhang, "Experimental demonstration of an acoustic magnifying hyperlens," Nat. Mater. 8, 931-934 (2009).

${ }^{8}$ S. Zhang, L. Yin, and N. Fang, "Focusing ultrasound with an acoustic metamaterial network," Phys. Rev. Lett. 102, 194301 (2009).

${ }^{9}$ S. Cummer and D. Schurig, "One path to acoustic cloaking," New J. Phys. 9, 45 (2007)

${ }^{10}$ B. Bonello, C. Charles, and F. Ganot, "Lamb waves in plates covered by a two-dimensional phononic film,” Appl. Phys. Lett. 90, 021909 (2007).

${ }^{11}$ F. Hsiao, A. Khelif, H. Moubchir, A. Choujaa, C. Chen, and V. Laude, "Waveguiding inside the complete band gap of a phononic crystal slab," Phys. Rev. E 76, 056601 (2007).

${ }^{12}$ A. Khelif, B. Aoubiza, S. Mohammadi, A. Adibi, and V. Laude, "Complete band gaps in two-dimensional phononic crystal slabs," Phys. Rev. E 74, 046610 (2006). 
${ }^{13}$ S. Mohammadi, A. Eftekhar, A. Khelif, W. Hunt, and A. Adibi, "Evidence of large high frequency complete phononic band gaps in silicon phononic crystal plates," Appl. Phys. Lett. 92, 221905-221905 (2008).

${ }^{14} \mathrm{~T}$. Wu, Z. Huang, T. Tsai, and T. Wu, "Evidence of complete band gap and resonances in a plate with periodic stubbed surface," Appl. Phys. Lett. 93, 111902 (2008).

${ }^{15}$ T.-C. Wu, T.-T. Wu, and J.-C. Hsu, "Waveguiding and frequency selection of lamb waves in a plate with a periodic stubbed surface," Phys. Rev. B 79, 104306 (2009).

${ }^{16}$ Y. Lai, Y. Wu, P. Sheng, and Z. Zhang, "Hybrid elastic solids," Nat. Mater. 10, 620-624 (2011)

${ }^{17} \mathrm{H}$. Huang and C. Sun, "Wave attenuation mechanism in an acoustic metamaterial with negative effective mass density," New J. Phys. 11, 013003 (2009).

${ }^{18}$ S. Yao, X. Zhou, and G. Hu, "Experimental study on negative effective mass in a 1d mass-spring system," New J. Phys. 10, 043020 (2008).

${ }^{19}$ T. Still, G. Gantzounis, D. Kiefer, G. Hellmann, R. Sainidou, G. Fytas, and N. Stefanou, "Collective hypersonic excitations in strongly multiple scattering colloids," Phys. Rev. Lett. 106, 175505 (2011).

${ }^{20} \mathrm{~J}$. Hsu and $\mathrm{T}$. Wu, "Lamb waves in binary locally resonant phononic plates with two-dimensional lattices," Appl. Phys. Lett. 90, 201904 (2007).

${ }^{21}$ M. Oudich, M. Senesi, M. B. Assouar, M. Ruzenne, J.-H. Sun, B. Vincent, Z. Hou, and T.-T. Wu, "Experimental evidence of locally resonant sonic band gap in two-dimensional phononic stubbed plates," Phys. Rev. B 84, 165136 (2011).
${ }^{22}$ G. Wang, X. Wen, J. Wen, L. Shao, and Y. Liu, "Two-dimensional locally resonant phononic crystals with binary structures," Phys. Rev. Lett. 93, 154302 (2004).

${ }^{23}$ Z. Cui, T. Chen, H. Chen, and Y. Su, "Experimental and calculated research on a large band gap constituting of tubes with periodic narrow slits," Appl. Acoustics 70, 1087-1093 (2009).

${ }^{24}$ M. Hirsekorn, P. Delsanto, A. Leung, and P. Matic, "Elastic wave propagation in locally resonant sonic material: Comparison between local interaction simulation approach and modal analysis," J. Appl. Phys. 99, 124912 (2006).

${ }^{25} \mathrm{~W}$. Xiao, G. Zeng, and Y. Cheng, "Flexural vibration band gaps in a thin plate containing a periodic array of hemmed discs," Appl. Acoustics 69, 255-261 (2008).

${ }^{26} \mathrm{~K}$. Bertoldi and M. Boyce, "Mechanically triggered transformations of phononic band gaps in periodic elastomeric structures," Phys. Rev. B 77, 052105 (2008).

${ }^{27}$ J. Vasseur, O. Matar, J. Robillard, A. Hladky-Hennion, and P. Deymier, "Band structures tunability of bulk $2 \mathrm{~d}$ phononic crystals made of magnetoelastic materials," AIP Adv. 1, 041904-041904 (2011).

${ }^{28} \mathrm{~L}$. Airoldi and M. Ruzzene, "Design of tunable acoustic metamaterials through periodic arrays of resonant shunted piezos," New J. Phys. 13, 113010 (2011).

${ }^{29}$ M. Ruzzene, "Frequency-wavenumber domain filtering for improved damage visualization," Smart Mater. Struct. 16, 2116 (2007).

${ }^{30}$ N. W. Hagood and A. von Flotow, "Damping of structural vibrations with piezoelectric materials and passive electrical networks," J. Sound Vib. 146, 243-268 (1991). 